\title{
Realizing long radio-frequency quadrupole accelerators with multiple shorter and independent cavities
}

\author{
Chuan Zhang $\oplus^{1,}{ }^{, *}$ Holger Podlech, ${ }^{2}$ and Eugene Tanke ${ }^{3}$ \\ ${ }^{1}$ GSI Helmholtz Center for Heavy Ion Research, Planckstraße 1, D-64291 Darmstadt, Germany \\ ${ }^{2}$ Institute for Applied Physics, Goethe University, Max-von-Laue-Straße 1, D-60438 Frankfurt a. M., Germany \\ ${ }^{3}$ European Spallation Source ERIC, Box 176, SE-22100 Lund, Sweden
}

(Received 13 December 2019; accepted 16 April 2020; published 27 April 2020)

\begin{abstract}
It is well known that the tuning of a long radio-frequency quadrupole accelerator is demanding. This study investigated how to realize efficient long RFQ accelerators with multiple shorter and independent cavities. From the rf point of view, the use of shorter cavities has many advantages, e.g., enlarged mode separation and simplified rf tuning, but the beam matching between cavities will become an issue, especially at high current and low energy. Taking a more than $9 \mathrm{~m}$ long, high current machine as an example, this paper presents the design concepts and methods leading to good beam quality and high beam transmission throughout a multistage RFQ accelerator.
\end{abstract}

DOI: 10.1103/PhysRevAccelBeams.23.042003

\section{INTRODUCTION}

Along with the development in the past 50 years, the radio-frequency quadrupole (RFQ) accelerator has become a kind of very important proton and ion accelerating structure either as a stand-alone machine or as an injector to a large facility. This structure is excellent in capturing, focusing and bunching a dc beam from an ion source and the low energy transport section, but is not very efficient for accelerating it to high beam energy. Typically, the output beam energy of an RFQ accelerator is lower than $3 \mathrm{AMeV}$, and its structure length is shorter than $4 \mathrm{~m}$.

For some projects (see Fig. 1), however, longer RFQs have been constructed or operated. Except for the ISAC RFQ [1] and the HSI ("high current injector" translated from German "hochstrominjektor") RFQ [2], they are all using the four-vane structure based on the $\mathrm{TE}_{210}$ mode. One important reason for having a long RFQ can be due to the accelerating structure behind the RFQ, usually a drift tube linac (DTL). The accelerating cells at the low energy end of a DTL need a reasonable length so that the tubes can be physically constructed and also can be easily cooled if the duty factor is high. Typically, the length of the first DTL cell should be longer than $3 \mathrm{~cm}$ in case no integrated quadrupole magnet is necessary for the tube; otherwise, the RFQ needs to provide an output beam with even higher

\footnotetext{
*c.zhang@gsi.de
}

Published by the American Physical Society under the terms of the Creative Commons Attribution 4.0 International license. Further distribution of this work must maintain attribution to the author(s) and the published article's title, journal citation, and DOI. beam velocity. Furthermore, for some very high current machines, higher output energy of the RFQ can make the beam dynamics of the downstream DTL less challenging.

Usually, two major aspects in tuning an RFQ are: (1) to keep a good frequency separation between the operating mode and the nearest unwanted mode for enhancing the longitudinal stability; (2) to stabilize the quadrupole mode from disturbing dipole components. For the $\mathrm{TE}_{210}$ mode, the sensitivity to tuning errors can be expressed as proportional to $(L / \lambda)^{2}$, where $L$ is the RFQ length and $\lambda$ is the free-space wavelength $[3,4]$. It can be seen that the longer the RFQ, the more difficult the tuning. The HSI RFQ uses the interdigital $\mathrm{H}$-mode $(\mathrm{IH})$ structure based on the $\mathrm{TE}_{110}$ mode. Similar to the four-vane structure, the magnetic field lines in the HSI RFQ are parallel to the beam axis and turning around at the two ends of the cavity.

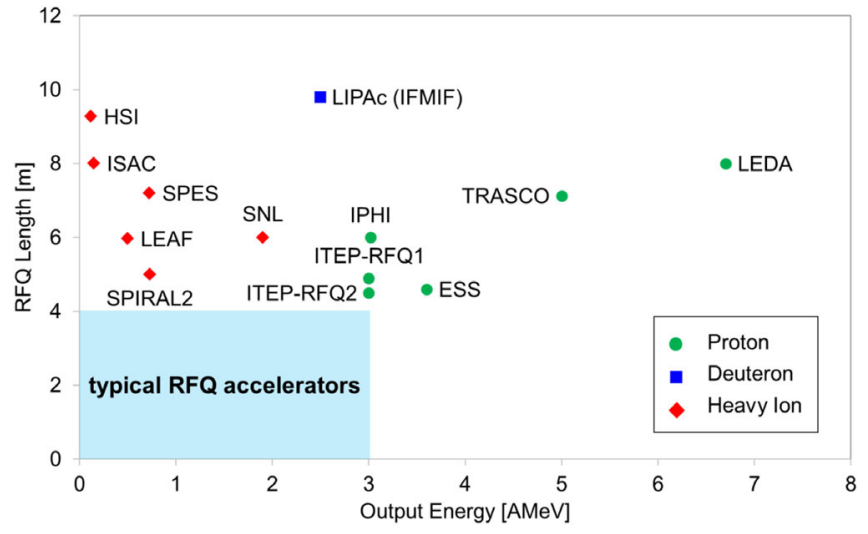

FIG. 1. Overview of some long ( $>4 \mathrm{~m}$ ) RFQ accelerators constructed or operated worldwide (note: the HSI and SPIRAL2 RFQs are also used for light ions). 
To address the difficulties in tuning long RFQs, several solutions have been proposed and developed. One is the socalled "resonant coupling" approach which divides a long RFQ accelerator into short (e.g., 1-2 m) sections and couples them together by short (a few mm) gaps between the end regions [5]. The $\mathrm{rf}$ simulation of a $4 \mathrm{~m}$ long, $425 \mathrm{MHz}$ RFQ shows that the frequency difference between the mode of interest and the neighboring mode can be increased from 1.9 to $6.6 \mathrm{MHz}$ via resonant coupling [5]. After the successful application to the $8 \mathrm{~m}$ long LEDA RFQ [6], this approach has been taken for many later long four-vane RFQs e.g., TRASCO [7] and IFMIF [8]. This kind of coupled structure needs only one rf power source and one beam dynamics design, so it is still one RFQ.

Another idea is to use a so-called "tandem-RFQ", i.e., two sequential RFQs with independent rf systems. Three examples are: (1) the constructed SNL RFQ with a total length $L_{\text {total }}=6 \mathrm{~m}$ [9]; (2) the constructed superconducting PIAVE RFQ with $L_{\text {total }}=\sim 2 \mathrm{~m}$ [10] (it is not a long RFQ, but uses a similar idea); (3) and the proposed normalconducting EURISOL RFQ with $L_{\text {total }}=7.8 \mathrm{~m}$ [11]. A challenge for using this concept is addressing the beam matching issue between the cavities, especially in the transverse phase spaces. Usually at the entrance to an RFQ accelerator, converging and similar particle distributions in both $x$ and $y$ planes are desired. But at the exit of an RFQ, typically the output beam is converging in one transverse plane and diverging in the other one. The matching problem can become very critical at high beam currents. The SNL and PIAVE projects run low beam currents in the order of $\mathrm{e} \mu \mathrm{A}$, so no special care is necessary between two separated cavities. EURISOL has been planned to work in two beam modes: heavy ion beams with negligible currents for continuous wave operation and up to $7.5 \mathrm{emA}$ light ion beams for pulsed operation [11]. In the EURISOL design, two RFQ structures are mounted inside the same tank and they are separated by a thin $\mathrm{rf}$ shielding wall (the gap between the two sets of electrodes is $1.7 \mathrm{~cm}$ long), thus accomplishing a short transition [11].

The motivation of this study is to investigate how to realize efficient long RFQs using multiple shorter and independent cavities with not only improved field stability and easy rf tuning but also high beam transmission and good beam quality, even at high currents. To introduce the design concepts and methods for fulfilling this purpose, the $9.3 \mathrm{~m}$ long HSI RFQ is adopted as an example by this study.

Serving as an injector to the UNILAC, the HSI RFQ can accelerate a wide variety of particle species from protons to uranium ions in the energy range of 2.2-120 AkeV. Some milestones of this machine are as follows: in 1996, the first design of the HSI RFQ for a $16.5 \mathrm{emA} \mathrm{U}^{4+}$ beam was started [12]; in 1998, the first HSI RFQ was constructed [2] (hereafter it is referred to as Version-1998); in 1999, the Version-1998 RFQ was put into operation; in 2004, the electrodes were replaced with new ones (with an improved
TABLE I. Main design parameters of the two constructed HSI RFQs.

\begin{tabular}{lcc}
\hline \hline & \multicolumn{2}{c}{ Version- } \\
& 1998 & 2008 \\
\hline Frequency $f[\mathrm{MHz}]$ & 36.136 & 36.136 \\
Input energy $W_{\text {in }}[\mathrm{AkeV}]$ & 2.2 & 2.2 \\
Output energy $W_{\text {out }}[\mathrm{AkeV}]$ & 120 & 120 \\
Design beam current $I[\mathrm{emA}]$ & 16.5 & 20 \\
Intervane voltage $V[\mathrm{kV}]$ & 125 & 155 \\
Midcell aperture $r_{0}[\mathrm{~mm}]$ & $5.2-7.8$ & $\sim 6.0$ \\
Maximum surface E-field $E_{s, \text { max }}[\mathrm{MV} / \mathrm{m}]$ & 31.8 & 31.2 \\
Number of cells & 357 & 409 \\
Total length $L_{\text {total }}[\mathrm{m}]$ (including the gaps & 9.27 & 9.27 \\
$\quad$ between the end plates and electrodes) & & \\
Input transverse emittance $[\pi$ mmmrad] & & \\
Unnormalized, total emittance $\varepsilon_{\text {in,unnorm,total }}$ & 138 & 210 \\
Normalized, rms emittance $\varepsilon_{\text {in,n,rms }}$ & 0.050 & 0.076 \\
Transmission $T$ [\%] & 89.5 & 88.5 \\
\hline \hline
\end{tabular}

radial matching section for a larger acceptance); in 2008, the second HSI RFQ was designed and produced for a $20 \mathrm{emA} \mathrm{U}^{4+}$ beam [13] (hereafter it is referred to as Version-2008) (for this upgrade, only the inner structure was changed, but the tank remained same); in 2009, the Version-2008 RFQ was put into operation; from 2009 until now, the second HSI RFQ is in routine operation (in 2019, the electrodes were renewed using the same design).

In the rf study performed for the first HSI RFQ [14], one can see that: (1) for the full-length tank, the frequency difference between the operating mode and the nearest mode is around $2 \mathrm{MHz}$; (2) for short tank segments, the higher resonant modes are by about a factor 8 above the fundamental mode.

Instead of using a nonconstant midcell aperture $r_{0}$ varied from 5.2 to $7.8 \mathrm{~mm}$ like the Version-1998 RFQ (see Table I), the Version-2008 adopted an almost constant $r_{0}$ $(\sim 6.0 \mathrm{~mm})$ [13] for the main RFQ, in order to keep the distributed capacitance along the accelerator nearly constant for easier tuning. This has no influence on the frequency gap for the mode separation (still $\sim 2 \mathrm{MHz}$ ). Another remarkable change in the Version-2008 design is that the intervane voltage $V$ was increased from 125 to $155 \mathrm{kV}$ [13]. On one side, it is because of a higher design beam current. On the other side, this is necessary for meeting the demand of having a constant $r_{0}$ for such a long machine. However, this change required more rf power (the power is proportional to $V^{2}$ ).

The design strategies for this study are as follows: (1) to increase the frequency gap for mode separation by using multiple shorter and independent cavities; (2) to apply almost constant but different $r_{0}$ values for different cavities, which eases tuning; (3) to adapt the intervane voltage $V$ individually according to the different space-charge situation in each cavity. 
This study has been performed in three steps: (1) the RFQ was divided into two pieces with drift space in between; (2) the RFQ was divided into two pieces with a medium energy beam transport (MEBT) section in between; (3) the RFQ was divided into three pieces with drift space in between.

The HSI RFQ covers the energy range of 2.2-120 AkeV. No matter a two-piece solution or a three-piece solution, therefore, the transition energy $W_{\text {trans }}$ between the cavities will be much lower than those of the three abovementioned tandem-RFQs (SNL: $W_{\text {trans }}=1.22 \mathrm{AMeV}$ [9]; PIAVE: $W_{\text {trans }}=341.7 \mathrm{AkeV}$ [10]; EURISOL: $W_{\text {trans }}=$ $260 \mathrm{AkeV}$ [11]). Furthermore, the much higher design beam current, $20 \mathrm{emA}$, will make the design work more challenging, so special measures are needed.

\section{2-RFQ SOLUTION WITH DRIFT SPACE BETWEEN CAVITIES}

First, a solution using two RFQ cavities with drift space in between has been investigated. Each cavity should have a structure length that is roughly half of the original RFQ length, i.e., $4.64 \mathrm{~m}$. Because the first cavity (RFQ 1) is more important for bunching and the second one (RFQ 2) is more important for acceleration, the transition energy has been chosen as $53.6 \mathrm{AkeV}$ which means that the RFQ 1 will cover an energy gain less than half of what is required for the whole HSI RFQ.

To reach good beam quality and high beam transmission, the design of the RFQ 1 is very important as well as relatively difficult, because it performs the main bunching at lower energy and sees stronger space-charge effects. Therefore, a so-called $\frac{\varepsilon_{l}}{\varepsilon}$ (ratio of the longitudinal and transverse emittances) $=1.0$ design approach [15] has been adopted for the RFQ 1, as it has been successfully applied for designing a $325 \mathrm{MHz}, 3 \mathrm{MeV}, 70-100 \mathrm{~mA}$ proton RFQ with high beam transmission, short structure length, and high beam quality. Combined with the new four-section procedure [16], this design approach takes advantage of the large resonance-free area provided by the $\frac{\varepsilon_{l}}{\varepsilon_{t}}=1.0$ condition for the beam evolution and can efficiently minimize emittance transfer and consequently reduce beam instabilities.

Typically an RFQ requires an input beam focused in both transverse planes $\left(\alpha_{\text {Twiss }}>0\right)$ and provides an output beam focused in one transverse plane $\left(\alpha_{\text {Twiss }}>0\right)$ and defocused in the other $\left(\alpha_{\text {Twiss }}<0\right)$. Therefore, it is challenging to achieve a good beam matching from the RFQ 1 to the RFQ 2. As a trade-off, a $3.2 \mathrm{~cm}$ long transition cell with no modulation has been added in front of the exit fringe-field region. With this cell, the beam energy is unchanged, but one obtains phase-space ellipses at the RFQ 1 output with Twiss parameter $\alpha_{\text {Twiss }} \cong 0$ in both transverse directions. This will make the matching of the beam into the second RFQ smoother.

The RFQ 2 will see an already bunched beam at the input. This is different from a normal RFQ which usually receives a continuous beam and needs to adapt it into a time varying field channel with a radial matching section (typically $4-6$ cells). Because the " $\alpha_{\text {Twiss }} \cong 0$ " method mentioned in the previous paragraph cannot provide an ideal matching, a traditional radial matchings section with large starting aperture, $-90^{\circ}$ synchronous phase, and no modulation has still been adopted for the RFQ 2. For a bunched beam, two cells are already sufficient for a good transition. Afterwards, the electrode aperture, the synchronous phase and the modulation parameter can be quickly changed to the values close to those at the RFQ 1 output, respectively.

Based on the above-mentioned methods, a $9.28 \mathrm{~m}$ long, $36.136 \mathrm{MHz}$ linac with two RFQ cavities (4.64 and $4.63 \mathrm{~m}$ long, respectively) and a drift space (drift length $d=1 \mathrm{~cm}$ ) in between has been designed. This solution is called " 2 RFQ with drift" in the following text. The evolution of the main design parameters along the accelerating channel is shown in Fig. 2, where $a$ is the minimum electrode

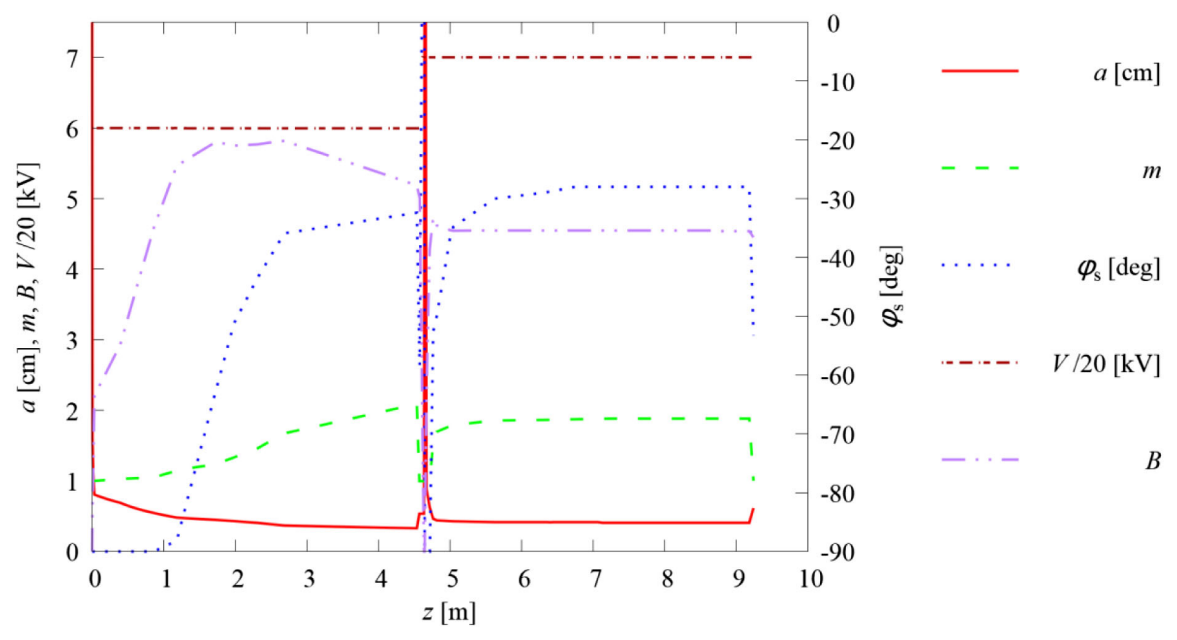

FIG. 2. Main design parameters of the "2-RFQ with drift" solution. 


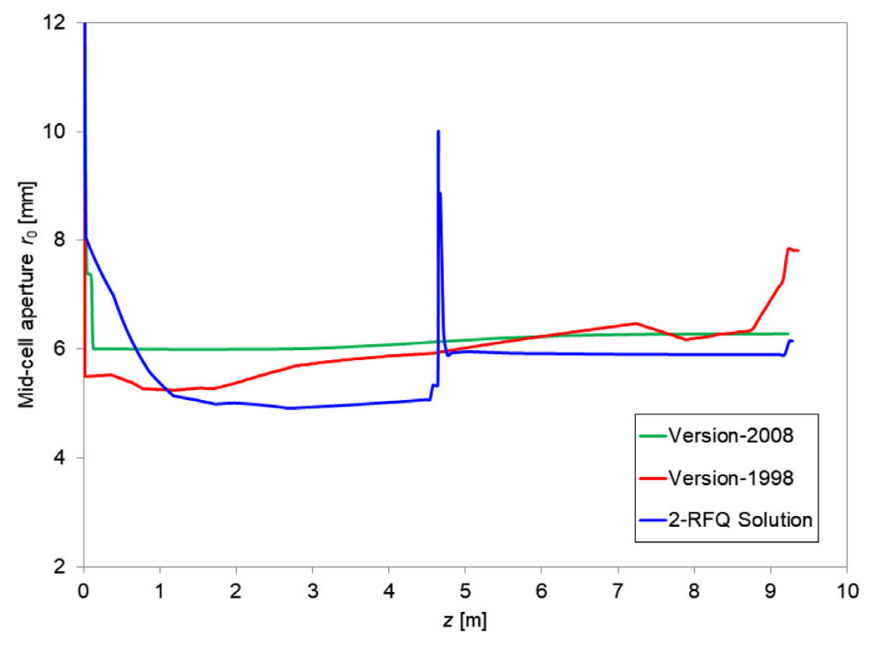

FIG. 3. Evolution of the midcell aperture along the accelerating channel (compared with the Version-1998 and Version-2008 RFQs).

aperture, $m$ is the electrode modulation, $\varphi_{s}$ is the synchronous phase, $V$ is the intervane voltage, and $B$ is the transverse focusing strength, respectively. It can be seen that: (1) the $V$ values have been set differently for the two cavities; (2) in the second cavity, all parameters are held almost constant, except some parameters, e.g., $\varphi_{s}$ have been changed shortly at the entrance and at the exit for better matching.

A comparison of the midcell aperture $r_{0}$ values between the new solution and the two existing designs is given in Fig. 3. Compared to the Version-1998 and Version-2008 RFQs, the 2-RFQ solution has a much bigger $r_{0}$ at the entrance, which helps increase the transverse acceptance as well as the beam transmission. Then its $r_{0}$ value becomes smaller than those of the other two designs, which supports using lower $V$ and saving rf power. Last but not least, the $r_{0}$ value is quasiconstant in the main part of the first cavity as well as in the whole second cavity, which is favorable for an easy rf tuning.

The beam dynamics simulation of the RFQs has been performed using the PARMTEQM code [17] developed by Los Alamos National Laboratory. The $20 \mathrm{emA} \mathrm{U}^{4+}$ input beam has a 4D-Waterbag distribution, and its transverse emittance is $210 \pi \mathrm{mm}$ mrad (unnormalized, total) or $0.076 \pi \mathrm{mm}$ mrad (normalized, rms), the same value as that used by the Version-2008 RFQ. The transverse beam envelopes for all particles as well as for $95 \%$ of the beam are shown in Fig. 4, where the black curves represent the midcell aperture of the RFQs and the beam hole aperture radius of the rf shielding wall between the RFQs. The total transmission of the linac is $96.05 \%$ and most losses happened between $z=1 \mathrm{~m}$ and $z=2 \mathrm{~m}$, so at low energy.

In Fig. 5, the transverse emittances are relatively constant as a function of the longitudinal position, while the longitudinal emittance for all particles increases between $z=2 \mathrm{~m}$ and $z=7 \mathrm{~m}$, but the longitudinal emittance curve for $99 \%$ of the beam indicates that this increase is due to only $\leq 1 \%$ of halo particles. It can be also seen that the design approach trying to hold $\frac{\varepsilon_{l}}{\varepsilon_{t}}=1.0$ successfully maintained the beam quality along the whole linac.

The output distribution of the RFQ 1 (see the top graphs of Fig. 6) shows that the introduced transition cell has successfully provided $\alpha_{\text {Twiss }} \cong 0$ transverse phase-space ellipses for entering into the RFQ 2. The beam distribution at the end of the whole linac (see the bottom graphs of Fig. 6) is still very concentrated. Its phase spread and energy spread for the main beam are well within $\pm 30^{\circ}$ and $\pm 1 \%$, respectively.

The above results are based on the 2-RFQ solution with a short drift between the two RFQ cavities (the drift length $d=1 \mathrm{~cm}$, and the distance between two sets of electrodes is $3.1 \mathrm{~cm}$ ). A batch of simulations has been performed by varying $d$ from 1 to $11 \mathrm{~cm}$ with an interval of $2 \mathrm{~cm}$.

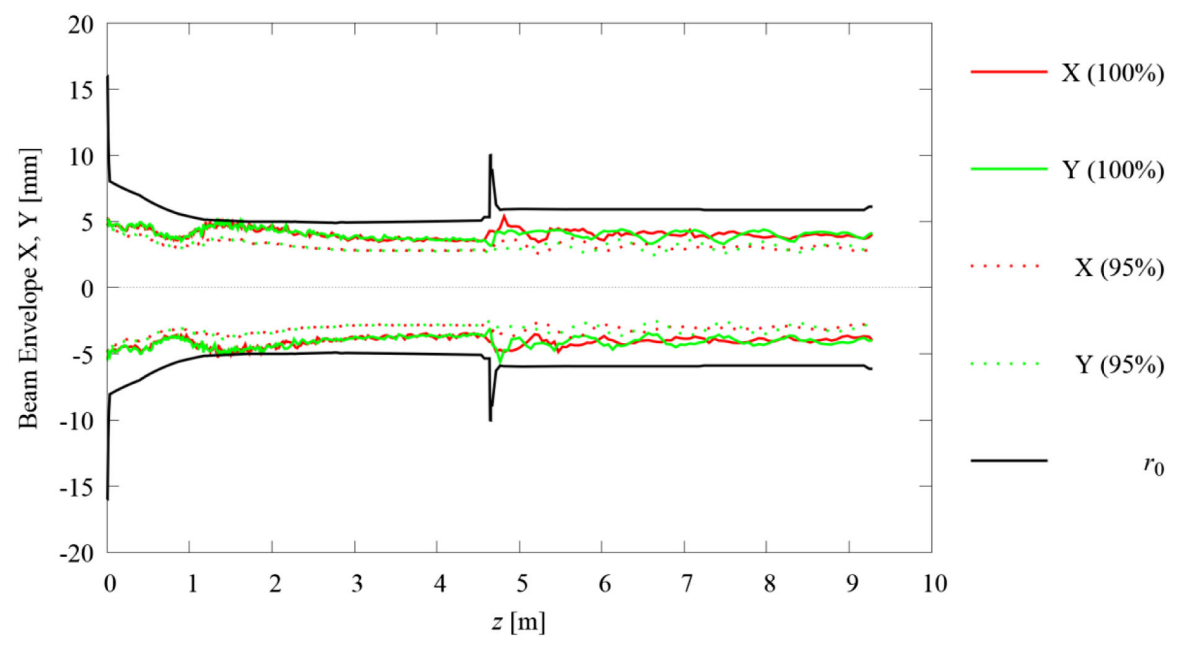

FIG. 4. Transverse beam envelopes for all particles as well as for $95 \%$ of the beam. 


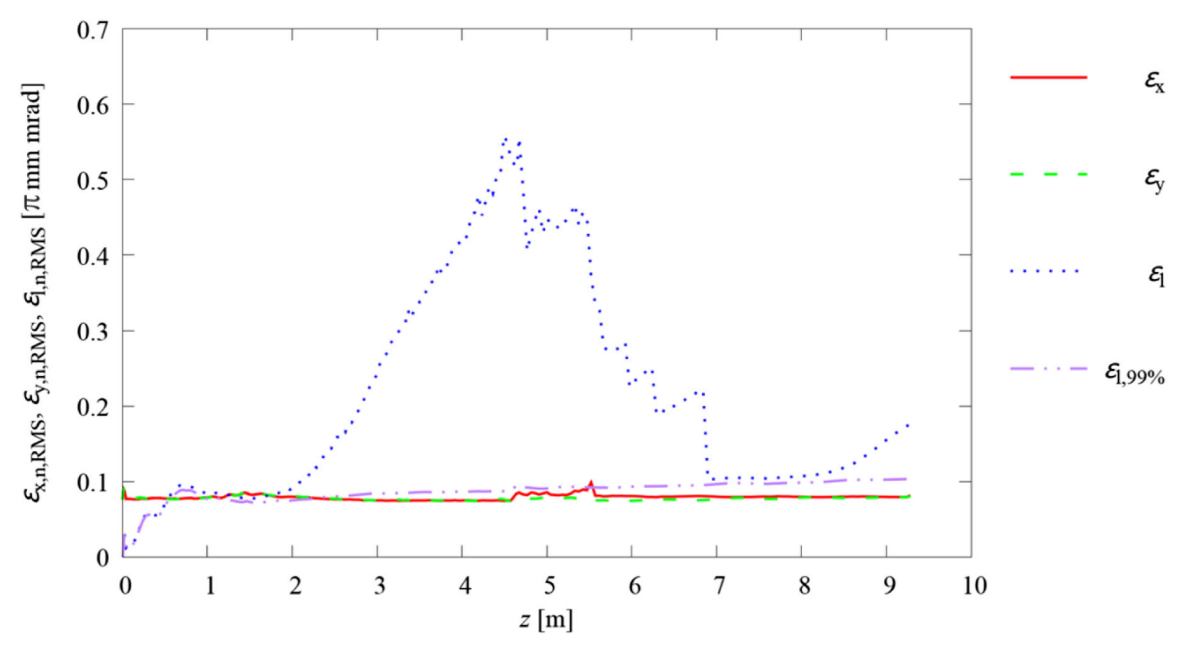

FIG. 5. Emittance evolutions along the linac (red, $x$ plane; green, $y$ plane; blue, longitudinal plane for all particles; purple, longitudinal plane for $99 \%$ of the beam).
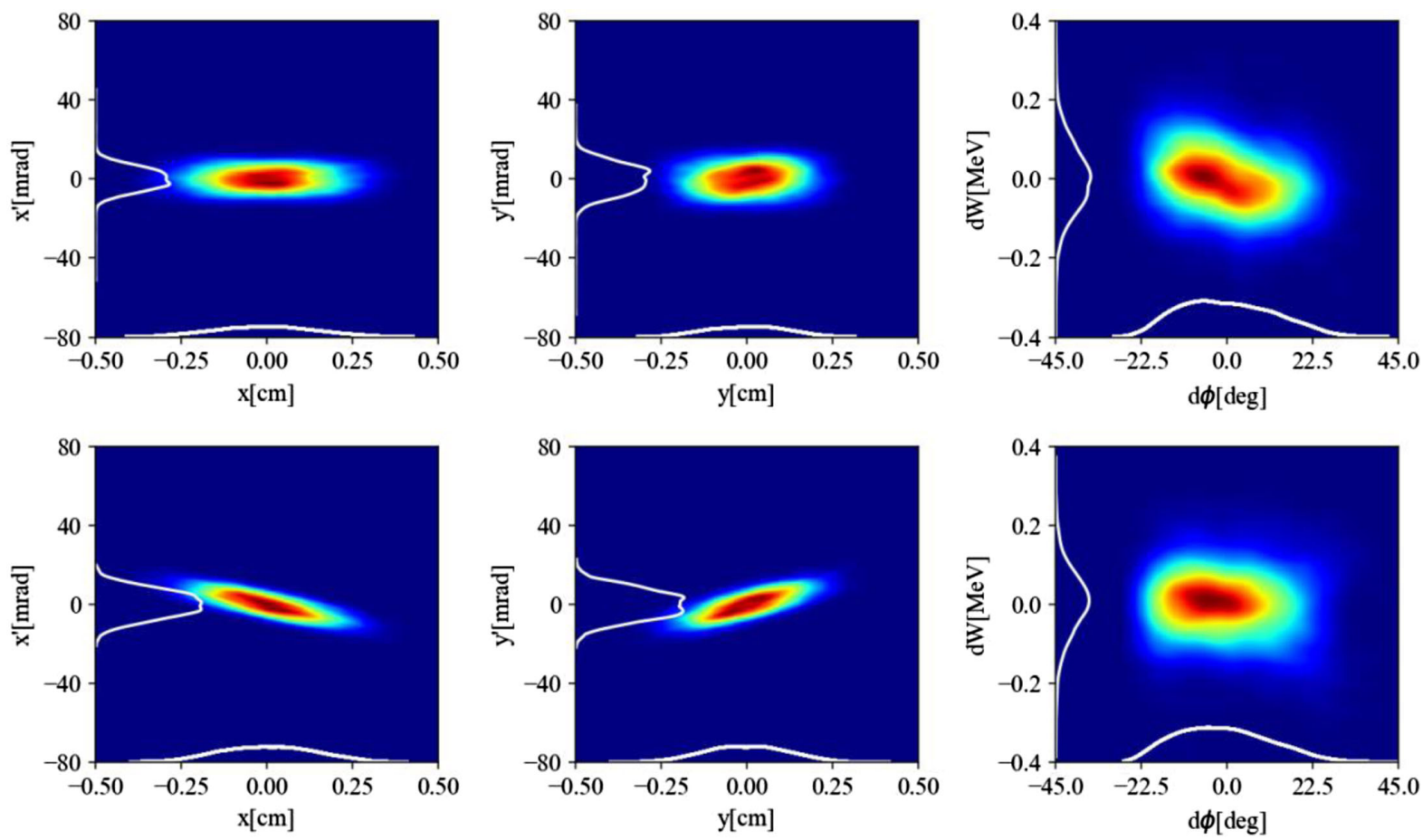

FIG. 6. Particle distributions at the RFQ 1 output (top graphs) and at the RFQ 2 output (bottom graphs).

Figure 7 shows that the beam transmission is still $~ 90 \%$ when $d=8 \mathrm{~cm}$. Figure 8 shows the evolution of the transverse and longitudinal emittances for different drift lengths. It can be seen that the change of the $d$ value has very little influence on both transverse emittance curves. Up to $z=7 \mathrm{~m}$, the evolutions of the longitudinal emittance values for the different cases are still very similar. Afterwards a larger $d$ leads to a larger longitudinal output emittance, e.g., $0.176 \pi \mathrm{mm} \mathrm{mrad}$ at $d=1 \mathrm{~cm}$ and $0.350 \pi \mathrm{mm} \mathrm{mrad}$ at $d=8 \mathrm{~cm}$. However, their $99 \%$ longitudinal output emittance values, $0.106 \pi \mathrm{mm} \mathrm{mrad}$ and $0.131 \pi \mathrm{mm} \mathrm{mrad}$ for $d=1$ and $8 \mathrm{~cm}$, respectively, are still relatively close.

Based on these results, different possible schemes can be applied to connect the two RFQ cavities whilst obtaining still reasonable transmission. One method is using a $1-5 \mathrm{~cm}$ thick wall to separate the rf fields of the two RFQs (see Fig. 9). Actually, it is also possible to have two individual end plates if $d \geq 4 \mathrm{~cm}$.

In case of $5 \mathrm{~cm} \leq d \leq 8 \mathrm{~cm}$, one beam diagnostic element, e.g., an ac current transformer (ACCT), can be 


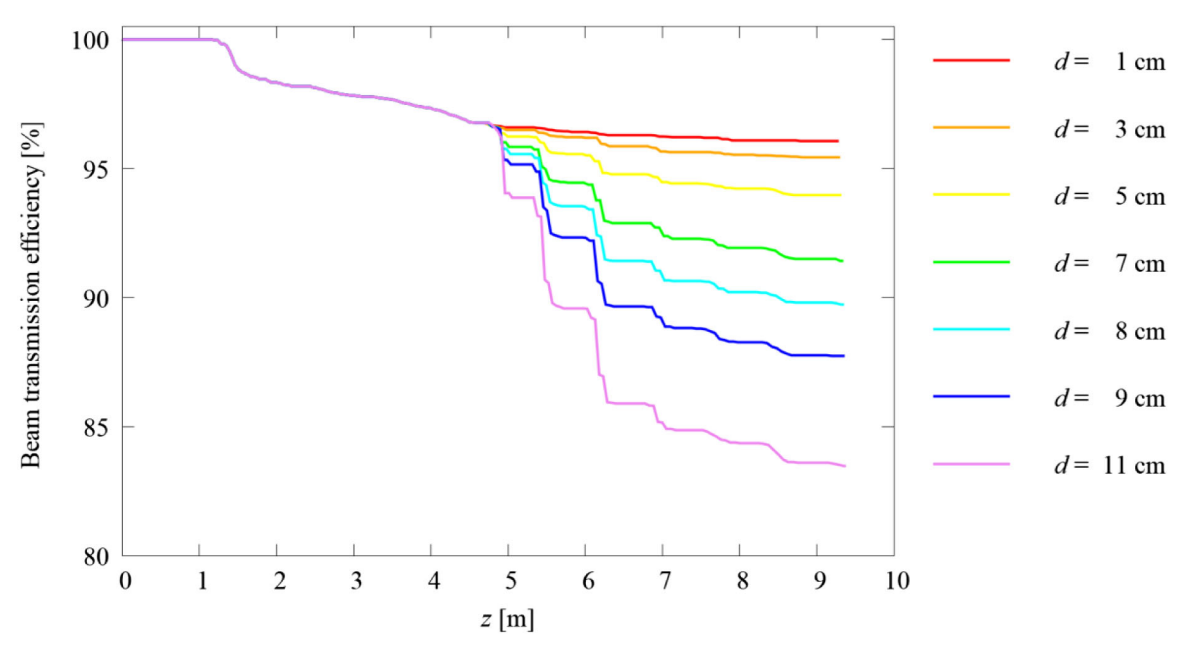

FIG. 7. Beam transmission curves for different drift lengths $(d=1-11 \mathrm{~cm})$.

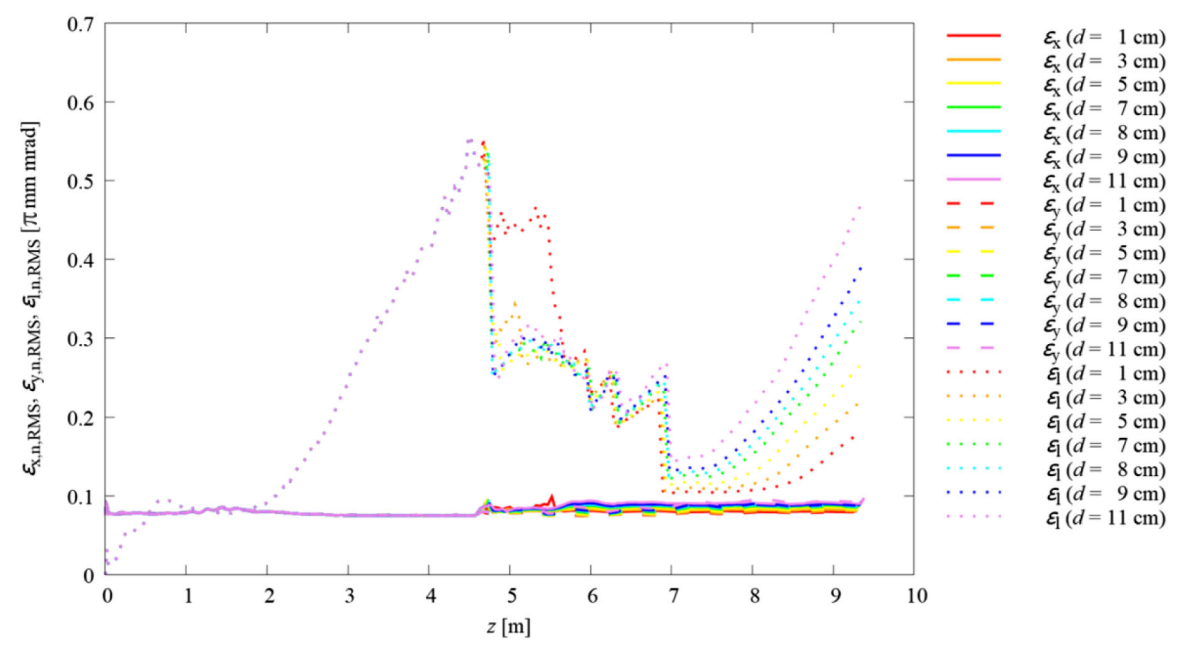

FIG. 8. Emittance evolution curves for different drift lengths $(d=1-11 \mathrm{~cm})$.

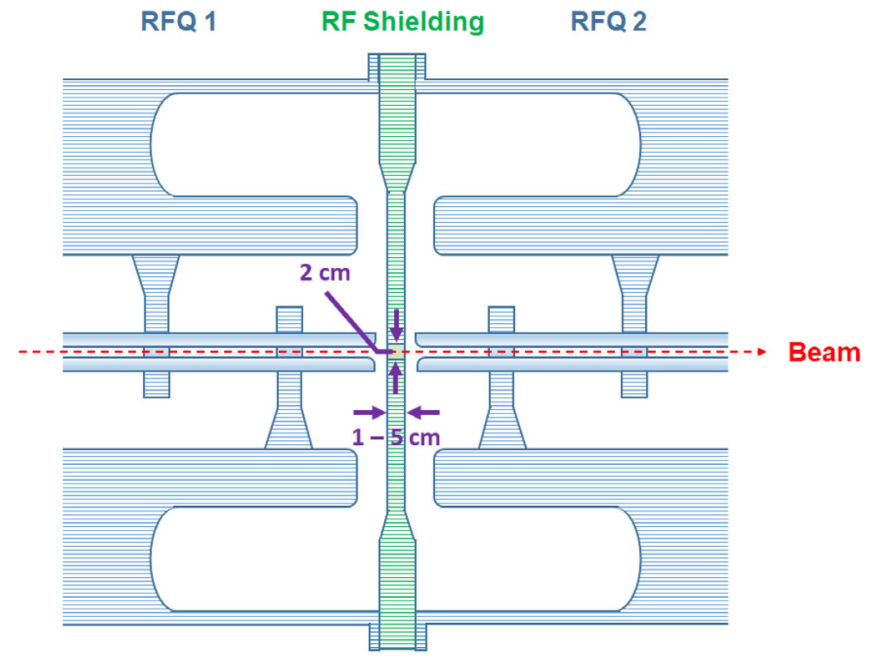

FIG. 9. Schematic plot for the connection of two RFQs by an rf shielding wall (the distance between two sets of electrodes excluding the $\mathrm{rf}$ shielding wall is $2.1 \mathrm{~cm}$ ).

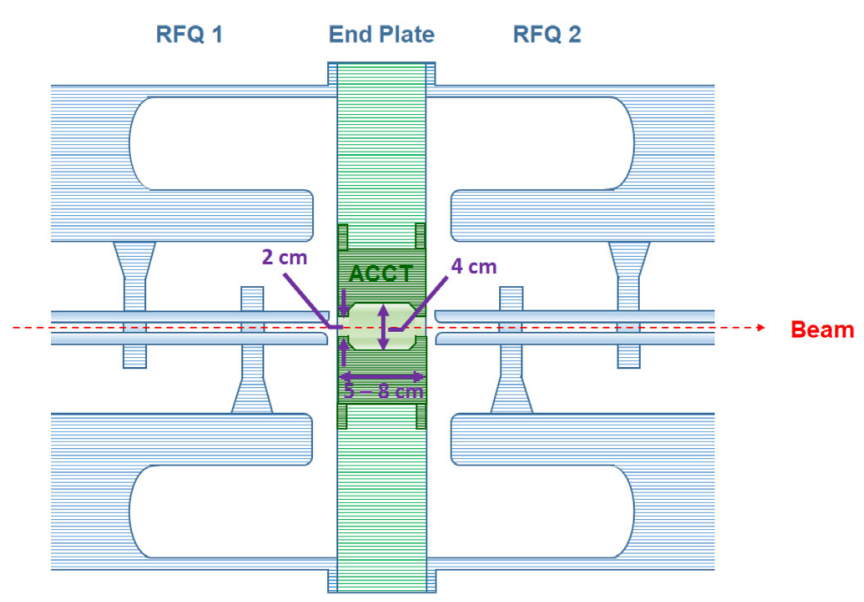

FIG. 10. Schematic plot of the connection between two RFQs for $5 \mathrm{~cm} \leq d \leq 8 \mathrm{~cm}$. 
introduced into this drift space and integrated with the end plate as shown in Fig. 10. The aperture diameter at the two ends of the added element can be held as $2 \mathrm{~cm}$ for good $\mathrm{rf}$ shielding and then enlarged to $4 \mathrm{~cm}$ in the middle.

\section{2-RFQ SOLUTION WITH MEBT BETWEEN CAVITIES}

To allow more intertank elements, e.g. XY-steerer and beam position monitor (BPM), for a better beam matching and tuning in the operation, the 2-RFQ solution has been further extended by including an MEBT section. As schematically shown in Fig. 11, the MEBT section consists of two $65 \mathrm{~cm}$ long triplets and one three-gap rebuncher cavity. At $36.136 \mathrm{MHz}$, a spiral structure [18] or a split-ring structure is a good candidate for the rebuncher. The main design parameters of the MEBT section are listed in Table II.
TABLE II. Main design parameters of the MEBT section.

\begin{tabular}{lccc}
\hline \hline & Triplet 1 & Rebuncher & Triplet 2 \\
\hline Quadrupole length [cm] & $16 / 20 / 16$ & & $16 / 20 / 16$ \\
Quadrupole inner & 3 & & 3 \\
$\quad$ diameter [cm] & & & $\leq 1.17$ \\
Quadrupole pole-tip field [T] & $\leq 1.14$ & & \\
Resonant frequency [MHz] & & 36.136 & \\
Period length [cm] & & 4.45 & \\
Effective gap voltage [kV] & 68 & \\
Tube inner diameter [cm] & & 2.4 & \\
\hline \hline
\end{tabular}

In this solution, the RFQ 1 is the same as that mentioned in Sec. II due to its good performance. The MEBT has been designed in a way that its output distribution should be close to the RFQ 1 output distribution so that the matching to the following RFQ cavity will be as smooth as possible.

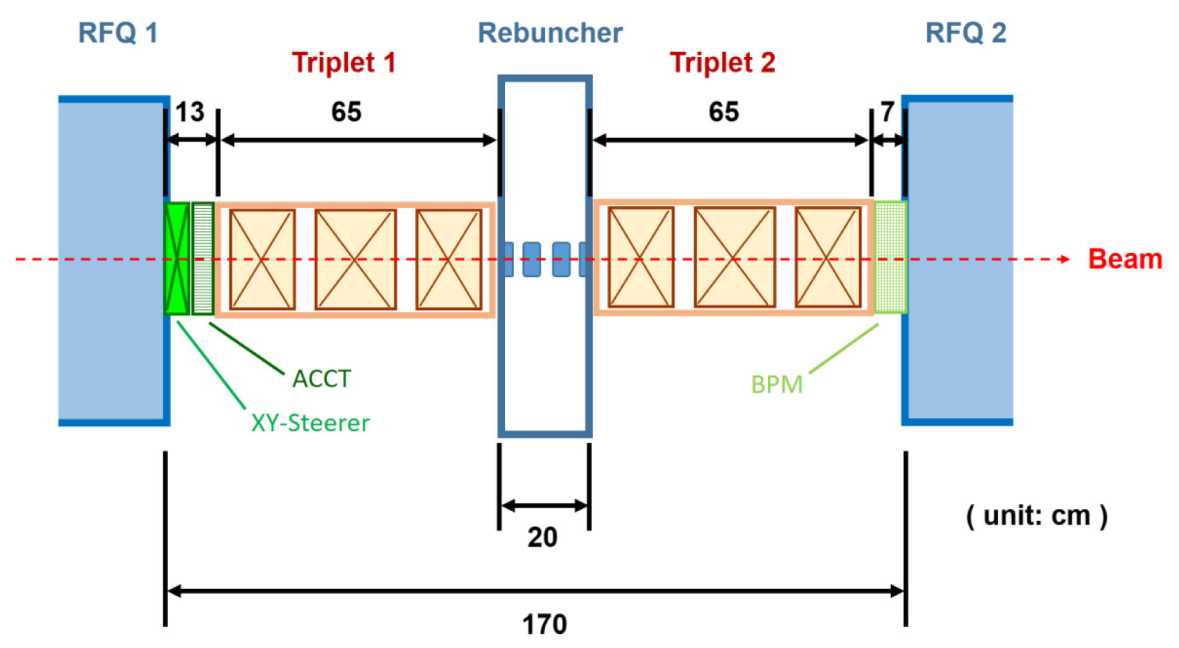

FIG. 11. Schematic plot of the connection between two RFQs with an MEBT.

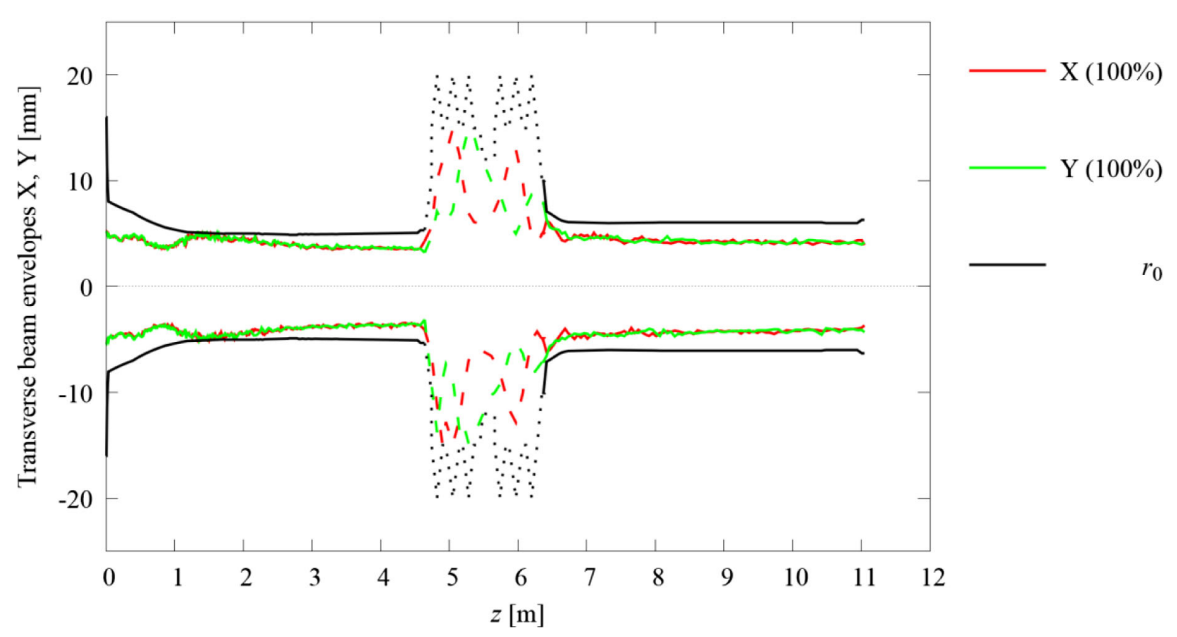

FIG. 12. Transverse beam envelopes for all particles along the linac (the solid lines are for the RFQs and the dashed lines are for the MEBT section, respectively). 


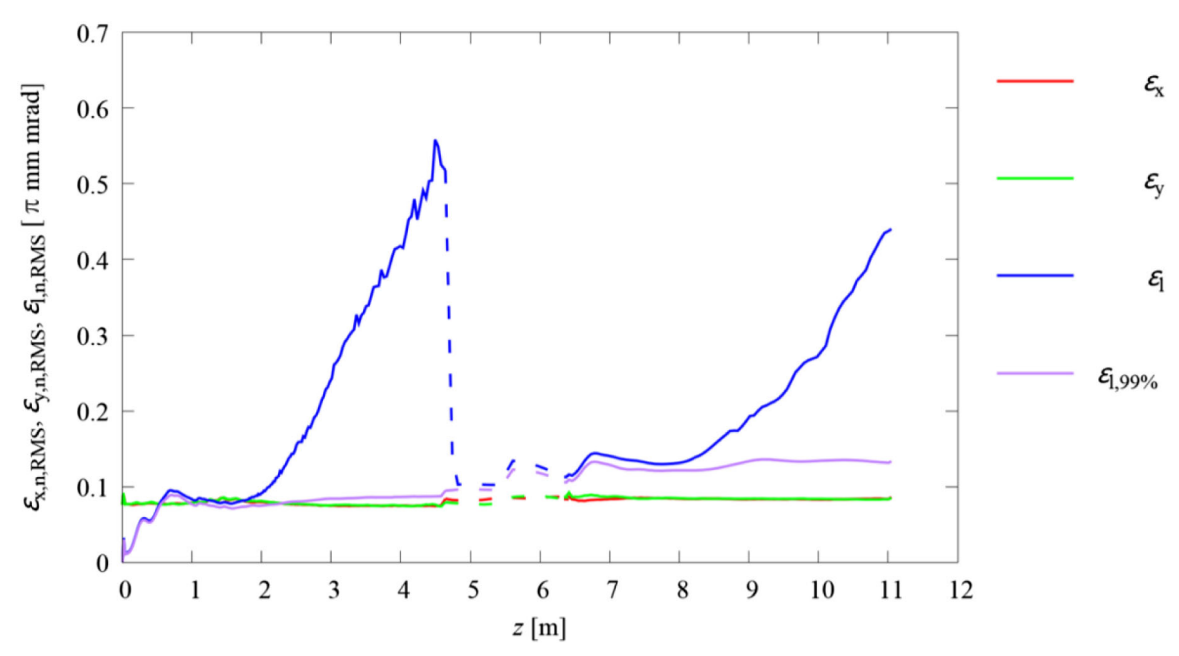

FIG. 13. Emittance evolutions along the linac with an MEBT (the solid lines for the RFQs, the dash lines for the MEBT section, the purple curve for $99 \%$ of the beam, respectively).

The RFQ 2 uses also the same design, except the starting part has been slightly adapted according to the differences caused by the MEBT.

Using the RFQ 1 output distribution (see the top graphs of Fig. 6) as the input distribution for the MEBT section, the DYNAC code [19] originally developed by CERN has been adopted for the beam dynamics simulation and the LORASR code [20] has been taken for a benchmarking. Both codes gave very similar results. Although the magnetic focusing for such a very low beta and very heavy ion beam is difficult, a $94.5 \%$ beam transmission has been achieved and the total transverse emittance growths are lower than
15\%. The transverse beam envelopes (see Fig. 12) have only some obvious oscillations in the MEBT part, but in the RFQ cavities they are still very similar to those of the "2-RFQ with drift" solution.

Figure 13 shows the transverse emittances are still relatively constant along the accelerating channel, despite the presence of the $1.7 \mathrm{~m}$ long MEBT. The longitudinal emittance has a little more growth with the new solution, but the $99 \%$ emittance curve is still flat.

Figure 14 shows the particle distributions at the MEBT exit and the whole linac exit. They are still comparable to the distributions shown in Fig. 6.
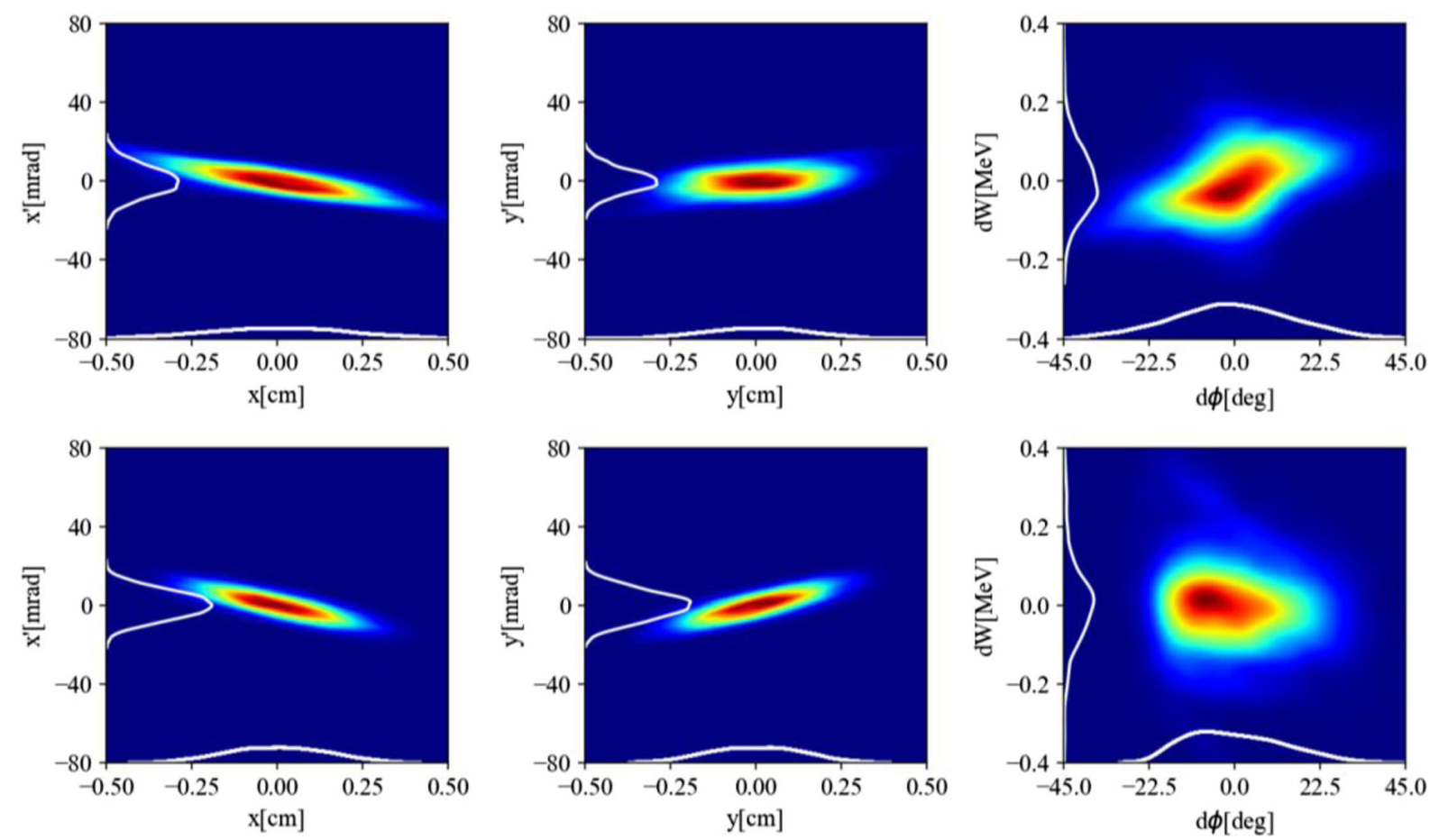

FIG. 14. Particle distributions at the MEBT output (top graphs) and at the RFQ 2 output (bottom graphs). 


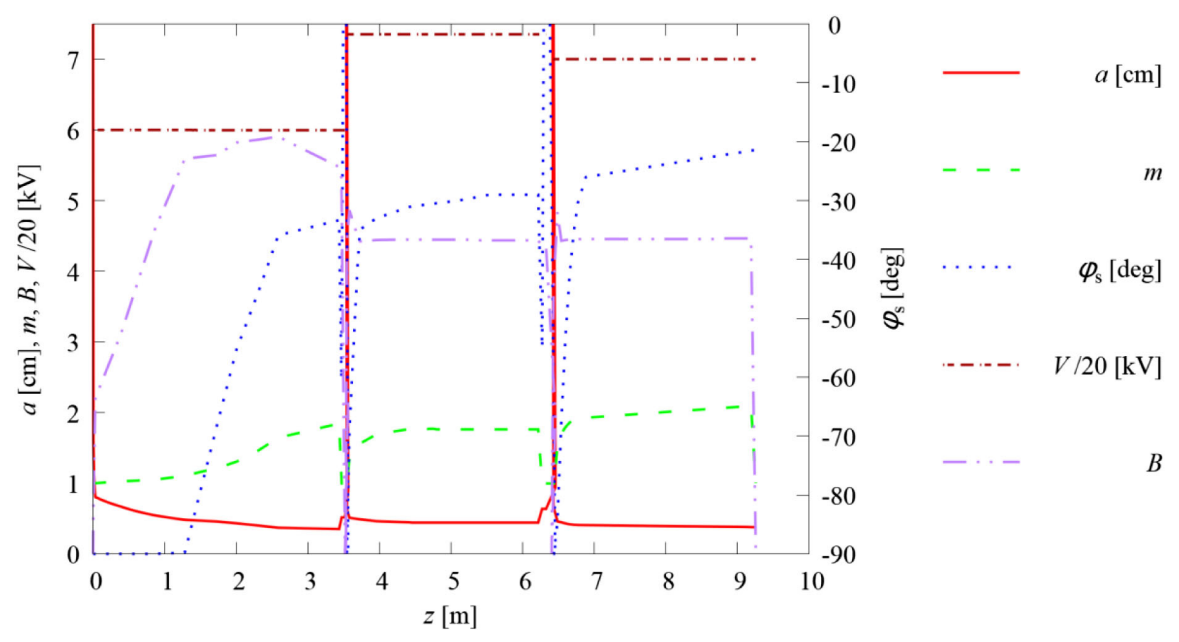

FIG. 15. Main design parameters of the "3-RFQ with drift" solution.

\section{3-RFQ SOLUTION WITH DRIFT SPACE BETWEEN CAVITIES}

Finally, a design using three RFQs with drifts in between has been made. The transition energy values between the cavities have been chosen as 32.6 and $78.5 \mathrm{AkeV}$, respectively. Due to the different output energy, the RFQ 1 design has been modified, but still following the $\frac{\varepsilon_{l}}{\varepsilon_{0}}=1.0$ design approach and the concept to have $\alpha_{\text {Twiss }} \cong 0$ output transverse phase-space ellipses. The main design parameters of the new linac are shown in Fig. 15, where the RFQ 1, the RFQ 2, and the RFQ 3 are 3.5, 2.9 and $2.9 \mathrm{~m}$ long, respectively. For this plot, the drift length between the cavities is $1 \mathrm{~cm}$. Generally speaking, the parameters are varying in a similar way to those in the 2-RFQ case (see Fig. 2).

The beam dynamics simulation of the "3-RFQ with drift" solution has also been performed using the PARMTEQM code.
As the RFQ 1 stops at even lower output energy, the beam dynamics of this linac is more challenging due to the space charge. Nevertheless, the total length is only $3 \mathrm{~cm}$ longer and the total transmission is only $1.6 \%$ lower, compared with the "2-RFQ with drift" case. Further simulations show that the transmission is still $\sim 90 \%$ when $d$ is prolonged to $6 \mathrm{~cm}$ (without modification and optimization of the RFQ 2 and the RFQ 3). A comparison of the main parameters between this solution and the 2-RFQ solutions is given in Table III.

From Table III, one can see that: (1) the maximum surface electric field for all these solutions is $30.9 \mathrm{MV} / \mathrm{m}$, lower than that of both the Version-1998 RFQ and the Version-2008 RFQ; (2) the intervane voltage for the solutions is between 120 and $147 \mathrm{kV}$, lower than $155 \mathrm{kV}$ used by the Version-2008 RFQ. These indicate that the new solutions can support a more reliable and more efficient (the power is proportional to $V^{2}$ ) operation.

TABLE III. A comparison of the 2-RFQ and 3-RFQ solutions (input emittance $=0.076 \pi \mathrm{mm} \mathrm{mrad)}$.

\begin{tabular}{|c|c|c|c|c|c|}
\hline \multirow[b]{2}{*}{ Number of RFQs } & \multicolumn{2}{|c|}{ 2-RFQ with drift } & \multirow{2}{*}{$\frac{\text { 2-RFQ with MEBT }}{2}$} & \multicolumn{2}{|c|}{ 3-RFQ with drift } \\
\hline & 2 & 2 & & 3 & 3 \\
\hline Transition between cavities & Drift & Drift & MEBT & Drift & Drift \\
\hline Transition length $d[\mathrm{~cm}]$ & 1 & 8 & 170 & 1 (each) & 6 (each) \\
\hline Maximum surface E-field $E_{s, \max }[\mathrm{MV} / \mathrm{m}]$ & 30.9 & 30.9 & 30.9 & 30.9 & 30.9 \\
\hline Intervane voltage $V[\mathrm{kV}]$ & \multicolumn{2}{|c|}{$120 / 140$} & $120 / 140$ & \multicolumn{2}{|c|}{$120 / 147 / 140$} \\
\hline Emittance growth in $x$ plane [\%] & & & & & \\
\hline For $100 \%$ beam & 7.9 & 21.1 & 13.2 & 13.2 & 23.7 \\
\hline For $99 \%$ beam & 3.9 & 15.8 & 9.2 & 9.2 & 18.4 \\
\hline \multicolumn{6}{|l|}{ Emittance growth in $y$ plane [\%] } \\
\hline For $100 \%$ beam & 6.6 & 14.5 & 11.8 & 10.5 & 23.7 \\
\hline For $99 \%$ beam & 2.6 & 10.5 & 7.9 & 6.6 & 18.4 \\
\hline \multicolumn{6}{|l|}{ Output emittance $\varepsilon_{1, \mathrm{n}, \mathrm{rms}}[\pi \mathrm{mm} \mathrm{mrad}]$} \\
\hline For $100 \%$ beam & 0.176 & 0.350 & 0.440 & 0.464 & 1.142 \\
\hline For $99 \%$ beam & 0.106 & 0.131 & 0.134 & 0.117 & 0.144 \\
\hline Total length $L[\mathrm{~m}]$ & 9.28 & 9.35 & 11.05 & 9.31 & 9.41 \\
\hline Total beam transmission $T$ [\%] & 96.1 & 89.7 & 94.5 & 94.5 & 89.3 \\
\hline
\end{tabular}




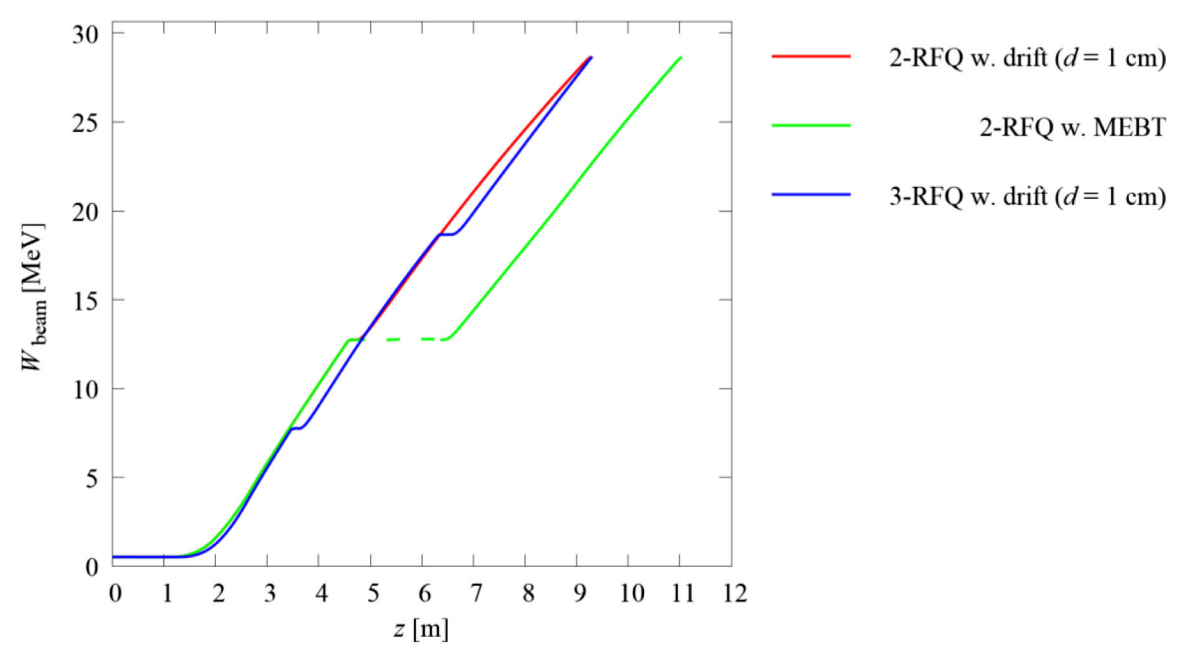

FIG. 16. Evolution of the synchronous energy for the three reference cases.

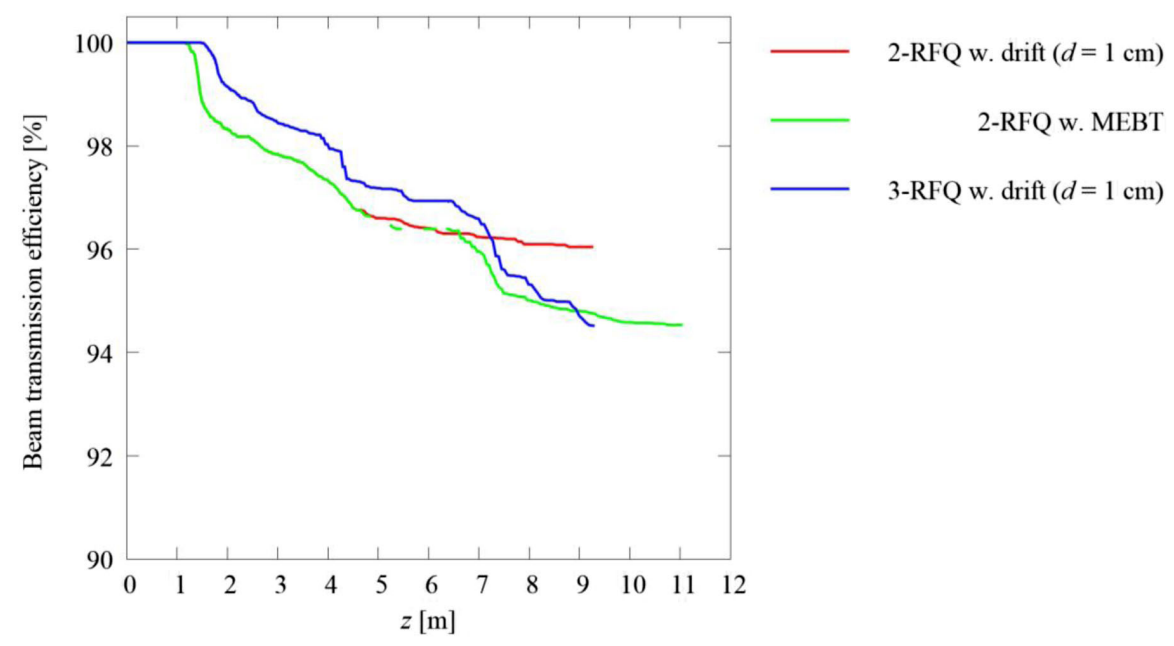

FIG. 17. Evolution of the beam transmission for the three reference cases.

In addition, the end-to-end evolution curves of synchronous energy and beam transmission efficiency are plotted in Figs. 16 and 17, respectively, for the three reference cases. Generally speaking, they are very comparable, if the MEBT is skipped.

\section{CONCLUSIONS}

Different scenarios using multiple shorter and independent cavities to realize a longer than $9 \mathrm{~m}$, low energy, and high current RFQ accelerator have been investigated. Compared to the single RFQ design, a solution using shorter cavities has the following advantages: it improves the longitudinal field stability and eases the rf tuning; one can use smaller and low-cost power amplifiers; it allows designing the different parts of the long accelerating channel individually and more efficiently-one can adapt the cavities to the changing beam situation along the beam line (for example, constant but different $r_{0}$ values can be applied for different cavities and also the intervane voltage of each cavity can be chosen more flexibly and reasonably); it supports to add beam diagnostics and knobs between cavities for a better beam matching and tuning in the operation.

Different from the resonant coupling approach that divides a long RFQ into several segments with short (a few mm long) gaps as transitions, which has only one radial matching section and one fringe field section, the multicavity approach used by this study has multiple radial matching sections and exit fringe field sections. Each cavity has to be treated separately, not only from an rf point of view but also in the sense of beam dynamics.

To solve the beam matching problem between cavities as well as to reach good beam quality and high transmission also at high current, the design of the first RFQ cavity is always most difficult. One key task for the RFQ 1 is to bunch the input beam at low energy, so it is very important to adopt the $\frac{\varepsilon_{l}}{\varepsilon_{t}}=1.0$ design approach which does minimize the emittance transfer between the longitudinal and 
transverse planes and reduce beam instability. In addition, providing $\alpha_{\text {Twiss }} \cong 0$ transverse phase-space ellipses to the downstream cavities can allow a smooth matching and help maintaining the beam quality as well.

Benefiting from these special methods, the new multicavity approach developed by this study cannot only improve the rf performance of the HSI RFQ e.g. larger mode separation, easier flatness tuning, lower sparking risk, and less power consumption, but also reach high beam transmission and good beam quality with a comparable total length.

\section{ACKNOWLEDGMENTS}

Special thanks go to R. Tiede, T. Sieber, H. Vormann, and $\mathrm{A}$. Bechtold for the valuable discussions with respect to the two constructed HSI RFQs and the realistic dimensions for possible intertank elements.

[1] R. E. Laxdal, RFQ-IH radioactive beam linac for ISAC, AIP Conf. Proc. 473, 546 (1999).

[2] U. Ratzinger, K. Kaspar, E. Malwitz, S. Minaev, and R. Tiede, The GSI $36 \mathrm{MHz}$ high-current IH-type RFQ and HIIF-relevant extensions, Nucl. Instrum. Methods Phys. Res., Sect. A 415, 281 (1998).

[3] L. M. Young, Tuning and stabilization of RFQ's, in Proceedings of LINAC1990 (JACoW, Albuquerque, New Mexico, 1990), p. 530.

[4] D. D. Armstrong, W. D. Cornelius, F. O. Purser, R. A. Jameson, and T. P. Wangler, RFQ development at Los Alamos, Report No. LA-UR-84-498, Los Alamos National Laboratory, 1984.

[5] M. J. Browman and L. M. Young, Coupled radio-frequency quadrupoles as compensated structures, in Proceedings of LINAC1990 (JACoW, Albuquerque, New Mexico, 1990), p. 70.

[6] L. Young, Operations of the LEDA resonantly coupled RFQ, in Proceedings of PAC2001 (JACoW, Chicago, USA, 2001), p. 509.

[7] A. Pisent, M. Comunian, A. Palmieri, G. V. Lamanna, and D. Barni, TRASCO RFQ, in Proceedings of LINAC2000 (JACoW, Monterey, California, USA, 2000), p. 902.

[8] E. Fagotti et al., Beam commissioning of the IFMIF EVEDA very high power RFQ, in Proceedings of
IPAC2018 (JACoW, Vancouver, BC, Canada, 2018), p. 2902.

[9] F. D. McDaniel, B. L. Doyle, P. Rossi, D. L. Buller, R. W. Hamm, and H. Schone, The tandem-RFQ linac booster at Sandia National Laboratories, AIP Conf. Proc. 680, 986 (2003).

[10] G. Bisoffi, A. M. Porcellato, V. Andreev, G. Bassato, G. Bezzon, S. Canella, F. Chiurlotto, A. Lombardi, E. Chiaveri, W. Singer, T. Shirai, and S. Y. Stark, Superconducting RFQ's ready for ion beam operation at INFN-LNL, in Proceedings of EPAC2002 (JACoW, Paris, France, 2002), p. 266.

[11] A. Bechtold and H. Podlech, Proposal of a normal conducting CW-RFQ for the EURISOL post-accelerator and a dedicated -beam linac concept, in Proceedings of EPAC2006 (JACoW, Edinburgh, Scotland, United Kingdom, 2006), p. 1580.

[12] A. Schempp, Design of compact RFQ's, in Proceedings of LINAC1996 (JACoW, Geneva, Switzerland, 1996), p. 53.

[13] A. Kolomiets, S. Minaev, W. Barth, L. Dahl, H. Vormann, and S. Yaramyshev, Upgrade of the unilac high current injector RFQ, in Proceedings of LINAC2008 (JACoW, Vancouver, BC, Canada, 2008), p. 136.

[14] K. Kaspar and U. Ratzinger, Design of the GSI $36 \mathrm{MHz}$ RFQ accelerator on the base of Mafia calculations, in Proceedings of EPAC1996 (JACoW, Sitges, Barcelona, Spain, 1996), p. 1973.

[15] C. Zhang and H. Podlech, Design approach for a $325 \mathrm{MHz}$, $3 \mathrm{MeV}, 70-100 \mathrm{~mA}$ proton radio-frequency quadrupole accelerator with low emittance transfer, Nucl. Instrum. Methods Phys. Res., Sect. A 947, 162756 (2019).

[16] C. Zhang and A. Schempp, Beam dynamics studies on a $200 \mathrm{~mA}$ proton radio frequency quadrupole accelerator, Nucl. Instrum. Methods Phys. Res., Sect. A 586, 153 (2008).

[17] K. R. Crandall, T. P. Wangler, L. M. Young, J. H. Billen, G. H. Neuschaefer, and D. L. Schrage, RFQ Design Codes, Report No. LA-UR-96-1836, Los Alamos National Laboratory, 2005.

[18] A. K. Mitra and R. L. Poirier, High power test of the $35 \mathrm{MHz}$ spiral re-buncher cavity for the TRIUMF ISAC facility, in Proceedings of EPAC2000 (JACoW, Vienna, Austria, 2000), p. 1978.

[19] http://dynac.web.cern.ch/dynac/dynac.html.

[20] R. Tiede, G. Clemente, H. Podlech, U. Ratzinger, A. Sauer, and S. Minaev, LORASR code development, in Proceedings of EPAC2006 (JACoW, Edinburgh, Scotland, United Kingdom, 2006), p. 2194. 